\title{
Haptic Training Simulation
}

\author{
Arnaud Lelevé ${ }^{1 *}$, Troy McDaniel ${ }^{2}$ and Carlos Rossa ${ }^{3}$ \\ ${ }^{1}$ Univ Lyon, INSA Lyon, Université Claude Bernard Lyon 1, INSA Lyon, CNRS, Ampère UMR5005, Villeubanne, France, ${ }^{2}$ The \\ Polytechnic School, Arizona State University, Mesa, AZ, United States, ${ }^{3}$ Faculty of Engineering and Applied Science, Ontario \\ Tech University, Oshawa, ON, Canada
}

Immersive virtual environments combined with kinaesthetic and/or tactile haptic feedback are becoming an essential building block of simulator training in a variety of applications. This paper aims to illustrate the interest of hands-on training simulation with haptic feedback. We review the recent application domains and we expose the progress and open challenges in the medical domain which is particularly demonstrative. The paper then addresses two aspects of haptic feedback that could help enhance modern haptic training simulators' performance, namely transparent and efficient actuation for kinaesthetic feedback and tactile feedback. This research topic, beyond technological progress, should help design kinaesthetic and tactile haptic interfaces and motivate the use of new actuation techniques for more realistic and effective feedback in simulations as soon as users are immersed in virtual environments.

\section{OPEN ACCESS}

Edited by:

Stefania Serafin,

Aalborg University

Copenhagen, Denmark

Reviewed by:

Fabien Danieau,

InterDigital, France

Theodore Lim

Heriot-Watt University,

United Kingdom

*Correspondence:

Arnaud Lelevé

arnaud.leleve@insa-lyon.fr

Specialty section:

This article was submitted to

Technologies for VR,

a section of the journal

Frontiers in Virtual Reality

Received: 18 March 2020

Accepted: 01 June 2020

Published: 08 July 2020

Citation:

Lelevé A, McDaniel T and Rossa C

(2020) Haptic Training Simulation.

Front. Virtual Real. 1:3

doi: 10.3389/frvir.2020.00003
Keywords: haptics, hands-on training, simulation, tactile, kinaesthetic

\section{INTRODUCTION}

Hands-on training is necessary to acquire skills for interaction with one's environment. It is natural during our childhood (Fagard et al., 2016), it is widespread during our scholar education (Ma and Nickerson, 2006), and it is necessary to practice professions featuring manual tasks (surgeons, metal workers, plane pilots...) (Stevens and Weale, 2003). Using simulation for hands-on training has been widely used in numerous domains [in healthcare: Nestel et al. (2019) for instance], as training in real situations is not always possible for various reasons: possible harming of patients in Medicine, destructive, or dangerous experiments, costly material or unreachable environment (such as nuclear and deep-water operations). Computer-Based Simulation (CBS) has been a recent progress in this area, bringing new opportunities to train when it was not physically possible [see Edwards et al. (2020) in anesthesiology domain, for instance]. But the most recent upgrade came with the integration of haptic feedback to these CBS, providing users with force or tactile feedback. The word "haptic" comes from the Greek word "haptomai" (' $\alpha \tau \tau$ ' "touch." Human haptic senses gather kinaesthetic (force) and tactile feedback, two complementary senses enabling us to gather information from the environment during physical interactions (such as manipulation or walking). A haptic training simulator (HTS) is a CBS extended with force and/or tactile feedback. To illustrate in one sentence the progress and state of current HTS research: consider that haptic feedback delivered to users immersed in a Virtual Reality (VR) environment while interacting with virtual characters, alters their perception and physiological arousal within the virtual environment (Krogmeier et al., 2019). This paper aims at (1) illustrating the interest of hands-on training simulation with haptic devices, (2) depicting current research works by highlighting two specific topics (actuation in HTS and tactile feedback integration), and (3) reviewing recent progress and open challenges to propose future directions that can lead 
to their democratization. These topics have been chosen as they still suffer with scientific and technical important locks to be open to provide users with more realistic feedback and a better training simulation.

We recall, in Section main applications of haptic training simulators, the main application domains of such simulators, and we focus on medical applications, which are the most prominent ones in the literature. In Section discussion: challenges for better haptic training, we discuss effective actuation and tactile feedback. We then conclude and draw some future directions the research on HTS should follow.

\section{MAIN APPLICATIONS OF HAPTIC TRAINING SIMULATORS}

The first interest of HTS is for training on dangerous tasks in safe conditions, typically in the military field [for instance with firearm shooting training (Wei et al., 2019)]. One of the most well-known applications of training simulation is for plane piloting. Recent works (Biddle and Buck, 2019) also involved Ground Procedure training with a VR headset (to view the cockpit with earphones and microphone for audio feedback and verbal interaction) and a VR hand controller or haptic gloves to manipulate instruments in the cockpit (for life-like, veridical touch). They also have been applied for firefighting training where the difficulty (for trainees and HTS designers) is that real situations are always different: they require highly customizable simulators to be trustworthy (Nahavandi et al., 2019). Furthermore, even if not induced by dangerous situations, some various daily-life applications exist [haptic and interactive training for drawing and painting on 3D objects (Shima and Soga, 2019), learning piano (Gerry et al., 2019), ...], and as haptic technology enhances, new ones appear each year.

A second interest of HTS is for training on repetitive tasks which require efficiency, typically in the manufacturing field, that requires rapid hands-on training of operators due to a high turn-over and the lack of skilled low level qualified workers. The difficulty here is that the gestures to learn involve the synchronization of both hands, and therefore, the use of two synchronized haptic interfaces. Most widespread applications correspond to tasks of assembly (Abidi et al., 2019, Arbeláez et al., 2019, Garbaya et al., 2019, Li et al., 2019), disassembly (Yang et al., 2019), and also maintenance (Neges et al., 2018, Numfu et al., 2019) which are still nowadays particularly manually operated. Loch et al. reviewed the research about haptic training on manual manufacturing procedures in Loch et al. (2018). Some other HTS applications are found in soldering for non-robotized lowand medium- series (James et al., 2019) or operating overhead traveling cranes (Noda et al., 2019).

The third main interest of HTS is to prevent harm to patients in the medical field. This is the field most studied in the literature, before manufacturing applications. This is why it is more detailed here. Surgeons have to perform difficult medical operations requiring to cut, suture, etc. Before performing such dexterous gestures, and even throughout their work life, to stay current they require hands-on training. Traditional medical simulators such as cadavers and animals have been used for decades in medical universities, but for ethical and cost reasons, phantoms (physical models made with non-biological materials) are used more often. Yet, phantoms focus on a limited range of classical cases and wear down, which requires frequent change of their worn parts, becoming costly long-term: this generates further increasing costs. Their limited realness and availability in universities and hospitals lead to medical student populations training on necessary gestures on patients during their first internships. This has progressively become less acceptable while, over the last decade, Virtual Reality (VR) simulators progressively appeared [see Kyaw et al. (2019) for a recent review about this topic] to overcome the aforementioned limitations. Such simulators can be parametrized to reproduce many more situations than phantoms. They also enable objective assessment through tangible recorded data analysis of trainees' achievements. VR simulators have been progressively improved to offer more realistic environments in $2 \mathrm{D}$, and in immersive 3D (Adams et al., 2019), and with haptic feedback (HTS). The didactic interest of HTS has been demonstrated in Panait et al. (2009) for advanced laparoscopy tasks, for suturing applications (Talasaz et al., 2017) in the context of robotic-assisted surgery, but also in Sainsbury et al. (2020) for percutaneous nephrolithotomy training. It is demonstrated that haptic feedback helps users manipulate their instruments better, and reduces tissue damage and accidental hits. However, simulation shall never completely replace training in real situations; it only delays the moment of application on real patients, for their own security. Also, medical simulators are nowadays limited by the inability to fully capture the complexity of human body anatomy in a mathematical model. The more the simulation is realistic, the more computer resources it requires along with accurate biomechanical modeling and programming. The same conclusion applies to the other aforementioned application domains. Haptic actuators still require some active research as they are an important link in the haptic feedback chain. Next section discusses kinaesthetic and tactile actuation.

\section{DISCUSSION: CHALLENGES FOR BETTER HAPTIC TRAINING}

The interest of haptic feedback in many applications has been demonstrated in the previous section. Yet, two interesting challenges have been selected to enhance the short and long term performance of such haptic training systems: (1) How different kinaesthetic actuation techniques can be used to improve simulation realism, and (2) how to introduce tactile stimulation to kinaesthetic haptic training simulations.

\section{Improving Realism Through Effective and Transparent Actuation}

Realism in haptic simulation requires both stability and transparency: stability is essential to correctly simulate a virtual environment and transparency can be considered one of the main benchmarks in kinaesthetic feedback. Mathematically, it may be defined as the ratio of the desired and simulated impedance, 
where the ideal ratio is unity for a given bandwidth (Rossa et al., 2014). The choice of the actuation system has strong implications in the fidelity of the haptic feedback and the way users perceive the virtual environment. There have been many attempts to improve haptic realism through effective and transparent kinaesthetic actuation techniques.

Electric motors: Traditionally, active actuators such as electric motors are the main power source in haptic devices. It is wellrecognized that due to quantization of time, position, and forces, any active haptic device is subjected to stability issues. Several authors have highlighted a critical tradeoff between the sampling rate, the virtual environment impedance, and energy dissipation due to viscous friction. To maintain stability, haptic devices bound their range of displayable impedance: the low-end being limited by the viscous friction required for energy dissipation, while the high-end is limited to the maximum control loop gain on the verge of the limit cycle of instability (Lacki et al., 2020). To overcome these limitations and improve simulation realism, alternative actuation techniques may be considered in haptic simulators.

Brakes: Passive actuators such as brakes or dampers generate force feedback by dissipating kinetic energy and thus are intrinsically stable. Electrorheological (ER), magnetorheological (MR), eddy current, and particle brakes are the most common types of brakes used in haptic applications. MR and ER brakes make use of a liquid substance that changes its apparent viscosity under the influence of a magnetic or an electric field, respectively. They generate a wide range of impedance but suffer from a greater response time than electric motors (Rossa et al., 2013). Both MR and ER brakes have a higher torque-to-mass ratio than DC motors, which can lead to great haptic transparency. Unarguably, the biggest drawback of passive actuators is the fact that they can only generate forces that oppose the direction of the input velocity. As a result, controlling the direction of the output force in multi-degree-of-freedom devices is not trivial and not always feasible (Lacki and Rossa, 2019). Despite this limitation, they offer a great alternative to improve haptic realism.

Pneumatic actuators are another interesting alternative to electric motors. They use compressed gasses to create mechanical motion with a high power-to-weight ratio and large motion range. They typically have a low mass, and are backdrivable (Senac et al., 2019). These characteristics allow complex, multidegree-of-freedom kinematic structures to be realized in a relatively compact package making them well-suited for haptic devices (Sugar et al., 2007). Notorious drawbacks of pneumatic actuators include the need for sources of compressed air and their poor efficiency. The efficiency of a pneumatic system is only $20 \%$ than that of a hydraulic or electrical system (Du et al., 2018). Nonlinearities in both force and airflow dynamics in pneumatic systems contribute to difficulties in controlling these actuators (Wolbrecht et al., 2010) making them more challenging for use in applications requiring high force precision such as in haptic simulation.

Hydraulic actuators create mechanical motion from fluids. Hydraulic actuators can generate the largest amount of force per weight and volume compared to the actuation methods presented above, with the drawback of having to include a power source in the design, limiting mobility. There are some exceptions to this condition; for example, the system in Umemura et al. (2009) can provide up to $89 \mathrm{Nm}$ of torque in a package that weighs only $4.5 \mathrm{~kg}$. Other advantages include smooth movements, lack of backlash, and satisfactory position tracking. However, they suffer from a slow response time compared to the actuation methods discussed earlier.

Series electric actuators (SEA) are another class of actuation technique that introduces an elastic element between the mechanical drive and the end effector making otherwise rigid structures compliant to input forces. Topologically, SEAs are more amenable to accurate force control than classical actuation techniques as the elastic element may be used to provide a direct force estimate (DeBoon et al., 2019). The addition of an elastic element in a haptic device may be counterintuitive, but comes with several advantages over rigidly-coupled actuation topologies such as improved shock tolerances, force control, stability, and efficiency. SEAs have proved to be an excellent foundation for force feedback devices as they also allow for decoupled actuator inertia and reduced frictional effects (Conti and Oussama, 2009). Additionally, SEAs under closedloop force control can achieve good force bandwidth with a low output impedance. Generally, the maximum stiffness the actuator can render is limited by the stiffness of the elastic element, constituting the main limitation of SEA for haptic rendering.

Hybrid actuators combining two or more distinct actuation techniques are also an efficient way of achieving high performance in haptic devices and cope with the limitations described earlier. Several hybrid actuators have been proposed including rotary knobs for vehicle control (Chapuis et al., 2006), surgical devices (Gonenc and Gurocak, 2012), and telerobotics systems (Walker et al., 2009). A typical hybrid actuator comprises an active element for energy restitution and a passive actuator to increase torque capability and dissipate energy as needed. From a control perspective, three main approaches can be distinguished. The first uses the active actuator to display the reference forces obtained from the virtual environment, while the passive actuator provides for the interaction stability by applying a controllable damping. In the second method, the passive actuator displays the virtual environment forces, while the active actuator compensates for friction in the mechanical structure, hence lowering the minimum achievable impedance and improving transparency. Alternatively, as in Rossa et al. (2014), both actuators can be combined to display reflective forces simultaneously.

Figure 1 shows different actuation arrangements that have been proposed in the literature as an attempt to improve haptic realism. Hybrid actuators combining different actuation sources are arguably the most robust solution for haptic applications that may eventually lead to the development of ultimately transparent haptic devices for hands-on training purposes.

\section{Introducing Tactile Feedback}

To illustrate the considerations for implementation of tactile stimulation within training, the scenario of telesurgery can be 

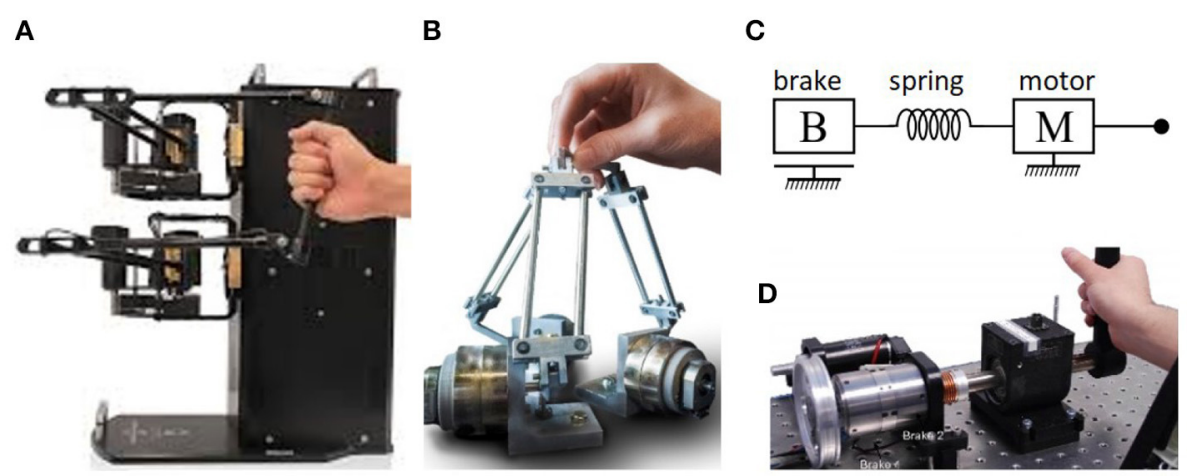

FIGURE 1 | Different actuation techniques for haptic devices. (A) is a traditional haptic device (HD² from Quanser Inc) with electric motors [reproduced from Quanser (2020) with permission from Quanser Inc.], (B) employs a set of three rheological brakes (Lacki and Rossa, 2020) [reproduced from Lacki and Rossa (2020) with permission from IEEE], in (C) a brake-motor-spring drive is proposed as an alternative to passive actuators (Conti and Oussama, 2009), and arrangement (D) has two unidirectional brakes coupled with a motor (Rossa et al., 2014) [reproduced from Rossa et al. (2014) with permission from IEEE].

observed as an exemplary domain. It has been noted that tactile feedback is present during minimally invasive surgery (MIS) and is provided not only in direct contact during palpation but also through surgical instruments (Bholat et al., 1999). Yet tactile feedback is largely missing in simulations for telesurgery and medical applications. Investigations have shown that training systems are in dire need of haptic stimulation to improve learning, sometimes even more so than visual information (Norkhairani et al., 2011). For exploration in haptic training, four classifications exist: surface texture determination through lateral motion, firmness exploration through pressure, form determination through contour feeling, and enclosure of the target for volume estimation (Klatzky et al., 1985). Their relative importance in the design of a tactile stimulation mechanism depends on the application domain; in surgical training, all four can be significant. Qualities of good tactile stimulation include (Overtoom et al., 2019): that it can sustain high output, has low inertia/friction, is highly responsive, and has a narrow bandwidth range. In general, the Degrees of Freedom (DOF) of the stimulation should match the task for the highest fidelity (fidelity being the level of resemblance to the real thing). Some studies note, however, that DOF, representational complexity, and relative emphasis of haptic feedback over other available modalities during training rely mainly on the complexity of the task and the experience level of the trainee (Pinzon et al., 2016).

Uniform distribution of force is a desirable mechanism when the role of tactile feedback is to effectively actuate the deformation of surfaces in training (Culjat et al., 2008). The ability of a stimulation medium to represent the objects, environment, and task characteristics in training scenarios also characterizes its effectiveness. Because the force application scenarios in domains such as surgical training are so variable, a flexible approach with high representational variability is extremely desirable. For instance, organs, tissues, bone structures, and blood vessels each have their own levels of resistance and fragility which should be differentiated within the display (Hamza-Lup et al., 2019). Often, since the task environment being simulated in training involves complex multi-modal information, the haptic feedback should be integrated with other feedback mediums such as visual or audio information in such a way that it more accurately reflects the characteristics of a real environment (Weik et al., 2019). This realism is also augmented by ensuring that the instrument used in haptic simulation matches the real instrument as closely as possible in properties such as mass. However, when high-fidelity representation is difficult or infeasible, tactile stimulation can instead be used to represent augmentative features which are only possible within a virtual training scenario (Okamura, 2009).

Rendering of training objectives and interactive surfaces in haptic space is another consideration. In many task environments, interference phenomena are present, and introduce noise into the haptic feedback received while completing the task. Systems intended to provide realistic training for these task scenarios should account for their existence. For example, in surgical environments, breathing, heartbeat, and waves of blood pressure all introduce noise into the surface feedback received when performing an operation. Therefore, the haptic approximation model within the training environment should be designed to either filter out or augment these effects (Hooshiar et al., 2020). While medical applications were chosen to demonstrate progress and open challenges related to integrating tactile stimulation into haptic training, the aim is to show the need for and potential impact of veridical interactions and feedback toward improving training simulations more broadly.

\section{CONCLUSION}

In this paper, we provided a short overview of typical application domains where haptic training simulation is required and often already effective. However, the (bio)mechanical modeling required precision, the computer resource limitations, the lack of efficient and compact actuators, and the subnominal integration of kinaesthetic, and tactile types of feedback, all together 
limit their practicality, which restricts their use to first stages of training. This is reinforced by the synchronization issues with sound and visual feedback. Recent progress in actuation technology has enhanced the compactness and the performances of haptic interfaces to provide finer kinaesthetic and tactile feedback. Therefore, the minimization of transmission delays in haptic (pressure, vibration, forces, and motion) feedback data delivery is of utmost importance to improve reaction time to contacts with surfaces, allowing the trainee to stop motion immediately upon this contact (Kontarinis and Howe, 1995).

This topic of research, beyond technological progress, should help design kinaesthetic and tactile haptic interfaces and motivate the use of new actuation techniques for more realistic and effective feedback in training simulations. Also, as motivation plays a crucial role in training in general and even more in motor learning and neurorehabilitation, haptic training associated with VR has great potential to enhance motivation during motor training (Bernardoni et al., 2019). Future directions include the

\section{REFERENCES}

Abidi, M. H., Al-Ahmari, A., Ahmad, A., Ameen, W., Alkhalefah, H. (2019). Assessment of virtual reality-based manufacturing assembly training system. Int. J. Adv. Manuf. Technol. 105, 3743-3759. doi: 10.1007/s00170-019-03801-3

Adams, H., Shinn, J., Morrel, W. G., Noble, J., and Bodenheimer, B. (2019). "Development and evaluation of an immersive virtual reality system for medical imaging of the ear," in Proc. of the international Conf. on SPIE Medical Imaging 2019, vol. 10951: Image-Guided Procedures, Robotic Interventions, and Modeling (San Diego, CA). doi: 10.1117/12.2506178

Arbeláez, J. C., Viganò, R., and Osorio-Gómez, G. (2019). Haptic Augmented Reality (HapticAR) for assembly guidance. Int. J. Interact. Des. Manuf. 13:673. doi: 10.1007/s12008-019-00532-3

Bernardoni, F., Özen, Ö., Buetler, K., and Marchal-Crespo, L. (2019). "Virtual reality environments and haptic strategies to enhance implicit learning and motivation in robot-assisted training," in Proc. of the 16th IEEE International Conference on Rehabilitation Robotics (ICORR 2019) (Toronto, ON), 760-765. doi: 10.1109/ICORR.2019.8779420

Bholat, O. S., Haluck, R. S., Murray, W. B., Gorman, P. J., and Krummel, T. M. (1999). Tactile feedback is present during minimally invasive surgery. J. Am. College Surgeons 189, 349-355. doi: 10.1016/S1072-7515(99)00184-2

Biddle, E., and Buck, B. (2019). "Adaptive team training for one," in Adaptive Instructional Systems. HCII 2019. Lecture Notes in Computer Science, eds R. Sottilare and J. Schwarz (Cham: Springer). doi: 10.1007/978-3-030-22341-0_2

Chapuis, D., Gassert, R., Burdet, E., and Bleuler, H. (2006). "Hybrid ultrasonic motor and electrorheological clutch system for MR-compatible haptic rendering," in 2006 IEEE/RSJ International Conference on Intelligent Robots and Systems (Beijing). doi: 10.1109/IROS.2006.282040

Conti, F., and Oussama, K. (2009). A new actuation approach for haptic interface design. Int. J. Robot. Res. 28.6, 834-848. doi: 10.1177/0278364908097958

Culjat, M., King, C., Franco, M., Bisley, J., Grundfest, W., and Dutson, E. (2008). Pneumatic balloon actuators for tactile feedback in robotic surgery. Indus. Robot Int. J. 35, 449-455. doi: 10.1108/01439910810893617

DeBoon, B., Nokleby, S., La Delfa, N., and Rossa, C. (2019). "Differentiallyclutched series elastic actuator for robot-aided musculoskeletal rehabilitation," in Proc. of the International Conference on Robotics and Automation (ICRA 2019) (Montreal, QC), 1507-1513. doi: 10.1109/ICRA.2019.87 93586

Du, H., Xiong, W., Li, Q., and Wang, L. (2018). Energy efficiency control of pneumatic actuator systems through nonlinear dynamic optimization. J. Cleaner Prod. 184, 511-519. doi: 10.1016/j.jclepro.2018.02.117

Edwards, D. A., Lampotang, S., Mahoney, B.;, Minehart, R. D., and Pian-Smith, M. C. M. (2020). Computer- and Web-Based Simulators and Virtual Environments application in new domains (rehabilitation is currently a growing one), the integration of most recent progress in hybrid actuation to provide even more compact and performant actuators, enhancing complex surface tactile feedback, synchronized with the other means of feedback, and careful attention to network design to minimize delay, noise, or other hindrances to the delivery of precise tactile and haptic information. A further investigation of close interactions (bumps, virtual hugs, collision avoidance) with participants with various characteristics, would also provide new insights into physiological arousal, emotion, and perception due to haptic feedback.

\section{AUTHOR CONTRIBUTIONS}

$\mathrm{AL}$ wrote the introduction, the main application section, and the conclusion. CR provided the efficient actuation section. TM produced the tactile feedback section. All authors contributed to the article and approved the submitted version.
Comprehensive Healthcare Simulation: Anesthesiology (Cham: Springer International Publishing), 117-125. doi: 10.1007/978-3-030-26849-7_12

Fagard, J., Rat-Fischer, L., Esseily, R., Somogyi, E., and O’Regan, J. K. (2016). What does it take for an infant to learn how to use a tool by observation? Front. Psychol. 7:267. doi: 10.3389/fpsyg.2016.00267

Garbaya, S., Romano, D., and Hattar, G. (2019). Gamification of assembly planning in virtual environment. Assembly Automation 39, 931-943. doi: 10.1108/AA-10-2018-0147

Gerry, L., Dahl, S., and Serafin, S. (2019). “ADEPT: exploring the design, pedagogy, and analysis of a mixed reality application for piano training," in Proc. of 16th Sound and Music Computing Conference (SMC 2019) (Malaga), 241-249.

Gonenc, B., and Gurocak, H. (2012). "Haptic interface with hybrid actuator for virtual needle insertion and tissue cutting," in Proc. of the IEEE Haptics Symposium (HAPTICS 2012) (Vancouver, BC). doi: 10.1109/HAPTIC.2012.6183830

Hamza-Lup, F. G., Bogdan, C. M., Popovici, D. M., and Costea, O. D. (2019). A survey of visuo-haptic simulation in surgical training. arXiv:1903.03272 [cs].

Hooshiar, A., Najarian, S., and Dargahi, J. (2020). Haptic telerobotic cardiovascular intervention: a review of approaches, methods, and future perspectives. IEEE Rev. Biomed. Eng. 13, 32-50. doi: 10.1109/RBME.2019.2907458

James, J., Rao, R. B., and Neamtu, G. (2019). Design of a bi-manual haptic interface for skill acquisition in surface mount device soldering. Soldering Surface Mount Technol. 31, 133-142. doi: 10.1108/SSMT-01-2018-0001

Klatzky, R. L., Lederman, S. J., and Metzger, V. A. (1985). Identifying objects by touch: an "expert system". Perception Psychophys. 37, 299-302. doi: 10.3758/BF03211351

Kontarinis, D. A., and Howe, R. D. (1995). Tactile display of vibratory information in teleoperation and virtual environments. Presence Teleoperat. Virtual Environ. 4, 387-402. doi: 10.1162/pres.1995.4.4.387

Krogmeier, C., Mousas, C., and Whittinghill, D. (2019). Human-virtual character interaction: toward understanding the influence of haptic feedback. Comput. Anim. Virtual Worlds 30:e1883. doi: 10.1002/cav.1883

Kyaw, B. M., Saxena, N., Posadzki, P., Vseteckova, J., Nikolaou, C. K., George, P. P., et al. (2019). Virtual reality for health professions education: systematic review and meta-analysis by the digital health education collaboration. J. Med. Internet Res. 21:e12959. doi: 10.2196/12959

Lacki, M., DeBoon, B., and Rossa, C. (2020). Impact of kinematic structure on the force displayability of planar passive haptic devices. IEEE Trans. Haptics. 13, 219-225. doi: 10.1109/TOH.2020.2970906

Lacki, M., and Rossa, C. (2019). "On the feasibility of multi-degree-of-freedom haptic devices using passive actuators," in Proc. of the IEEE/RSJ International Conference on Intelligent Robots and Systems (IROS 2019) (Macau), 7288-7293. doi: 10.1109/IROS40897.2019.8968164 
Lacki, M., and Rossa, C. (2020). Design and control of a 3 degreeof-freedom parallel passive haptic device. IEEE Trans. Haptics. doi: 10.1109/TOH.2020.2983037. [Epub ahead of print].

Li, Z., Wang, J., Yan, Z., Wang, X., and Anwar, M. S. (2019). "An interactive virtual training system for assembly and disassembly based on precedence constraints," in Advances in Computer Graphics. CGI 2019. Lecture Notes in Computer Science, Vol. 11542, eds M. Gavrilova, J. Chang, N. Thalmann, E. Hitzer, and H. Ishikawa (Cham: Springer).

Loch, F., Böck, S., and Vogel-Heuser, B. (2018). Teaching styles of virtual training systems for industrial applications-a review of the literature. Interact. Design Architect. J. IxD® $A$ 38, 46-63. Available online at: http://www.mifav.uniroma2. it/inevent/events/idea2010/index.php? $=10 \& a=10 \&$ link=ToC_38_P\&link= 38_3_abstract

Ma, J., and Nickerson, J. V. (2006). Hands-on, simulated, and remote laboratories: a comparative literature review. ACM Comput. Surv. 38, 7-30. doi: $10.1145 / 1132960.1132961$

Nahavandi, S., Wei, L., Mullins, J., Fielding, M., Deshpande, S., Watson, M., et al. (2019). "Haptically-enabled VR-based immersive fire fighting training simulator," in Intelligent Computing. CompCom 2019. Advances in Intelligent Systems and Computing, vol 997, eds K. Arai, R. Bhatia, S. Kapoor (Cham: Springer). doi: 10.1007/978-3-030-22871-2_2

Neges, M., Adwernat, S., and Abramovici, M. (2018). Augmented virtuality for maintenance training simulation under various stress conditions. Proc. Manuf. 19, 171-178. doi: 10.1016/j.promfg.2018.01.024

Nestel, D., Scerbo, M. W., and Kardong-Edgren, S. E. (2019). A Contemporary History of Healthcare Simulation Research, Healthcare Simulation Research (Cham: Springer International Publishing) 9-14. doi: 10.1007/978-3-030-26837-4_2

Noda, Y., Hoshi, R., and Kaneshige, A. (2019). Training simulator for acquiring operational skill to operate overhead traveling crane while suppressing load sway, shock and vibration. Hindawi 2019:3060457. doi: 10.1155/2019/3060457

Norkhairani, A. R., Zaman, H. B., and Ahmad, A. (2011). "Simulation for laparoscopy surgery with haptic element for medical students in HUKM: a preliminary analysis," in Visual Informatics: Sustaining Research and Innovations (Berlin, Heidelberg), 125-138. doi: 10.1007/978-3-642-25191-7_13

Numfu, M., Riel, A., and Noel, F. (2019). Virtual reality based digital chain for maintenance training. Proc. CIRP 84, 1069-1074. doi: 10.1016/j.procir.2019.04.268

Okamura, A. M. (2009). Haptic feedback in robot-assisted minimally invasive surgery. Curr. Opin. Urol. 19, 102-107. doi: 10.1097/MOU.0b013e32831a478c

Overtoom, E. M., Horeman, T., Jansen, F.-W., Dankelman, J., and Schreuder, H. W. R. (2019). Haptic feedback, force feedback, and force-sensing in simulation training for laparoscopy: a systematic overview. J. Surgical Edu. 76, 242-261. doi: 10.1016/j.jsurg.2018.06.008

Panait, L., Akkary, E., Bell, R., Roberts, K., Dudrick, S., and Duffy, A. (2009). The role of haptic feedback in laparoscopic simulation training. J. Surgical Res. 156, 312-316. doi: 10.1016/j.jss.2009.04.018

Pinzon, D., Byrns, S., and Zheng, B. (2016). Prevailing trends in haptic feedback simulation for minimally invasive surgery. Surg Innov. 23, 415-421. doi: $10.1177 / 1553350616628680$

Quanser, Inc. (2020). The global standard in transformational engineering labs for Controls, Robotics, and Mechatronics, optimized for the academic setting. Available online at: https://www.quanser.com/ (accessed May 27, 2020).

Rossa, C., Jaegy, A., Lozada, J., and Micaelli, A. (2013). Design considerations for magnetorheological brakes. IEEE/ASME Trans. Mechatr.19, 1669-1680. doi: 10.1109/TMECH.2013.2291966
Rossa, C., Lozada, J., and Micaelli, A. (2014). Design and control of a dual unidirectional brake hybrid actuation system for haptic devices. IEEE Trans. Haptics 7, 442-453. doi: 10.1109/TOH.2014.2346501

Sainsbury, B., Lacki, M., Shahait, M., Goldenberg, M., Baghdadi, A., Cavuoto, L., et al. (2020). Evaluation of a virtual reality percutaneous nephrolithotomy (PCNL) surgical simulator. Front. Robot. AI Virtual Environ. 6:145. doi: 10.3389/frobt.2019.00145

Senac, T., Lelevé, A., Moreau, R., Krähenbühl, L., Sigwalt, F., and Bauer, C. (2019). Simulating a syringe behaviour using a pneumatic cylinder haptic interface. Contr. Eng. Practice Elsev. 90, 231-240. doi: 10.1016/j.conengprac.2019.07.005

Shima, H., and Soga, M. (2019). Development of an interactive training support system for drawing and painting skill on a $3 \mathrm{D}$ object with $\mathrm{AR}$ and haptic interface. Proc. Comput. Sci. 159, 2015-2023. doi: 10.1016/j.procs.2019.09.374

Stevens, P., and Weale, M. (2003). "Education and economic growth," in International Handbook on the Economics of Education, eds G. Johnes and J. Johnes. doi: 10.4337/9781845421694.00009

Sugar, T., He, J., Koeneman, E. J., Koeneman, J. B., Herman, R., Huang, H., et al. (2007). Design and control of rupert: a device for robotic upper extremity repetitive therapy. IEEE Trans. Neural Syst. Rehab. Eng. 15, 336-346. doi: 10.1109/TNSRE.2007.903903

Talasaz, A., Trejos, A.L., and Patel, R.V. (2017). The role of direct and visual force feedback in suturing using a 7-dof dual-arm teleoperated system. IEEE Trans. Haptics 10, 276-287. doi: 10.1109/TOH.2016.2616874

Umemura, A., Saito, Y., and Fujisaki, K. (2009). "A study on power-assisted rehabilitation robot arms operated by patient with upper limb disabilities," in Proc. of the IEEE International Conference on Rehabilitation Robotics (ICORR 2009), pp. 451-456. doi: 10.1109/ICORR.2009.5209512

Walker, D. S., Thoma, D. J., and Niemeyer, G. (2009). "Variable impedance magnetorheological clutch actuator and telerobotic implementation," in IEEE/RSJ International Conference on Intelligent Robots and Systems (IROS 2009) (St. Louis, MO), 2885-2891. doi: 10.1109/IROS.2009.5354689

Wei, L., Zhou, H., and Nahavandi, S. (2019). Haptically enabled simulation system for firearm shooting training. Virtual Reality 23, 217-228. doi: 10.1007/s10055-018-0349-0

Weik, D., Lorenz, M., Knopp, S., Pelliccia, L., Feierabend, S., Rotsch, C., et al. (2019). "Integrating Tactile Feedback in an Acetabular Reamer for Surgical VR-Training", in Proc. of the IEEE Conference on Virtual Reality and 3D User Interfaces (VR 2019) (Osaka), 1227-1228. doi: 10.1109/VR.2019.8798287

Wolbrecht, E. T., Reinkensmeyer, D. J., and Bobrow, J. E. (2010). Pneumatic control of robots for rehabilitation. Int. J. Robot. Res. 29, 23-38. doi: $10.1177 / 0278364909103787$

Yang, Y. F., Yang, P., Li, J., Zeng, F., Yang, M., Wang, R., et al. (2019). Research on virtual haptic disassembly platform considering disassembly process. Neurocomputing 348, 74-81. doi: 10.1016/j.neucom.2018.05.120

Conflict of Interest: The authors declare that the research was conducted in the absence of any commercial or financial relationships that could be construed as a potential conflict of interest.

Copyright (c) 2020 Lelevé, McDaniel and Rossa. This is an open-access article distributed under the terms of the Creative Commons Attribution License (CC BY). The use, distribution or reproduction in other forums is permitted, provided the original author(s) and the copyright owner(s) are credited and that the original publication in this journal is cited, in accordance with accepted academic practice. No use, distribution or reproduction is permitted which does not comply with these terms. 